\title{
The murine bronchopulmonary microcirculation in hapten-induced inflammation
}

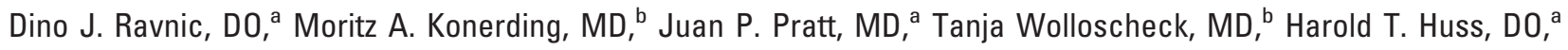 \\ and Steven J. Mentzer, MD ${ }^{a}$
}

From the Division of Thoracic Surgery, Brigham \& Women's Hospital, Boston, Mass, ${ }^{a}$ and the Department of Anatomy, Johannes Gutenberg University, Mainz, Germany. ${ }^{b}$

Supported in part by National Institutes of Health grants HL47078 and HL75426.

Read at the Eighty-sixth Annual Meeting of The American Association for Thoracic Surgery, Philadelphia, Pa, April 29-May 3, 2006.

Received for publication April 27, 2006; revisions received July 12, 2006; accepted for publication Aug 7, 2006.

Address for reprints: Steven J. Mentzer, MD, Room 259, Division of Thoracic Surgery, Brigham \& Women's Hospital, 75 Francis St, Boston MA 02115 (E-mail: smentzer@partners.or).

J Thorac Cardiovasc Surg 2007;133:97-103 0022-5223/\$32.00

Copyright $(9) 2007$ by The American Association for Thoracic Surgery

doi:10.1016/j.jtcvs.2006.08.054
Objective: The clinical observation of central bronchial artery hypertrophy in chronic lung inflammation suggests the possibility that the bronchial circulation may also participate in adaptive responses in peripheral lung inflammation.

Methods: To investigate the potential role of the bronchial microcirculation in peripheral lung inflammation, we developed a murine model of lung inflammation using the intratracheal instillation of the peptide-hapten trinitrophenol in presensitized mice.

Results: Clinical parameters indicated a peak inflammatory response at 96 hours. Similarly, gross and microscopic evidence of inflammation was observed 96 hours after antigen instillation. Using a forced oscillation technique to probe peripheral lung mechanics at 96 hours, we detected no change in central airway resistance $(P>$ $.05)$, but a significant increase in peripheral tissue resistance $(P<.05)$. The structure of the bronchial circulation was investigated by microsphere occlusion of the pulmonary circulation and corrosion casting of the bronchial circulation. SEM of the bronchial artery casts demonstrated (1) the presence of the peripheral bronchial circulation in mice, (2) interconnections of the two systems in the distal bronchial arteries and at the level of alveolar capillaries, and (3) functional evidence of increased bronchial perfusion of alveolar capillaries during mononuclear inflammation.

Conclusion: These results suggest an important adaptive role of the bronchial circulation in pulmonary inflammation.

$\mathrm{T}$ he lung has two arterial blood supplies, the pulmonary and bronchial systems. The functional role of the pulmonary circulation is primarily related to gas exchange. The pulmonary circulation supplies approximately $97 \%$ of total blood flow to the lungs. The remaining 3\% is supplied by the bronchial circulation and is thought to contribute to the nourishment of the central structures. ${ }^{1}$

Inflammation presents a unique challenge to this dual system. Continued perfusion of the inflamed peripheral lung is important for the delivery of nutrients and inflammatory cells; however, leukocyte delivery via the pulmonary circulation risks ventilation-perfusion mismatching and the compromise of gas exchange. A potential role for the bronchial circulation during inflammation is the delivery of nutrients and inflammatory cells to the peripheral lung via oxygenated blood from the systemic circulation. This possibility would require the presence of peripheral interconnections between the pulmonary and bronchial circulations, as well as a mechanism to increase volumetric flow in inflammatory conditions. Whether these adaptive changes occur in the peripheral bronchial circulation is unknown.

In this report, we established a murine model of peripheral lung inflammation using the intratracheal instillation of the peptide-hapten trinitrobenzyl sulphonate. The model was used to demonstrate (1) the presence of a peripheral bronchial 


$$
\begin{aligned}
& \text { Abbreviations and Acronyms } \\
& \begin{aligned}
\mathrm{G} & =\text { tissue damping parameter } \\
\mathrm{H} & =\text { tissue elastance } \\
\text { PBS } & =\text { phosphate buffered saline } \\
\text { SEM } & =\text { scanning electron microscopy } \\
\text { TNBS } & =\text { trinitrobenzene sulfonate }
\end{aligned}
\end{aligned}
$$

circulation in mice, (2) interconnections of the two systems in the distal bronchial arteries and at the level of alveolar capillaries, and (3) functional evidence of increased bronchial perfusion of alveolar capillaries during mononuclear inflammation.

\section{Materials and Methods \\ Mice}

Balb/c mice (Jackson Laboratory, Bar Harbor, Maine), 25 to $33 \mathrm{~g}$, were used in all experiments. The care of the animals was consistent with guidelines of the American Association for Accreditation of Laboratory Animal Care (Bethesda, Md).

\section{Antigen Stimulation}

The mouse abdomen was sheared and cleansed with water. Twenty-four hours later, $36 \mu \mathrm{L}$ of a $2.5 \%$ trinitrochlorobenzene (ChemArt, Egling, Germany) solution was sprayed onto a 1.5-cm diameter circular PhastTransfer Filter Paper (Pharmacia, Upsala, Sweden) in a 4:1 acetone/olive oil mixture. The antigensoaked filter paper was applied to the sheared abdomen with Tegaderm (3M, St Paul, Minn) for 24 hours. On postsensitization day $6,15 \mu \mathrm{L}$ of a $2.5 \%$ trinitrobenzene sulfonate (TNBS) solution was administered via the trachea. In control mice, $15 \mu \mathrm{L}$ of PBS was instilled intratracheally for the challenge dose.

\section{Clinical Assessment of Pneumonitis}

Total body weight was assessed daily. Activity level, fur ruffling, and tachypnea were scored daily on a 0 (normal) to 2 (severe) scale.

\section{Hematoxylin and Eosin Histology}

After euthanasia, phosphate-buffered saline solution-diluted, Tissue Freezing Medium-inflated tissues were harvested and immediately processed by quick freezing or aldehyde fixation. Quickfrozen tissue was sliced into $4 \times 4 \times 4$-mm blocks, coated with Tissue Freezing Medium (Triangle Biomedical Sciences, Durham, NC) and placed in 15-mm cryomolds. The cryomolds were placed in liquid nitrogen-cooled 2-methyl butane followed by immersion in liquid nitrogen and storage at $-80^{\circ} \mathrm{C}$. The aldehyde-fixed tissue was stained in Harris hematoxylin (Harris Modified SH26-F00D; Fisher, Pittsburgh, Pa) for 2 minutes followed by sequential rinses including a brief acid rinse. The slides were counterstained with Eosin Y (0.5\% eosin, 50\% ethanol, Fisher) followed by mounting with Permount (Fisher).

\section{Pulmonary Mechanics}

The forced oscillation technique used by the flexiVent (SciReq, Montreal, Quebec, Canada) ventilator uses multifrequency si- nusoidal perturbations to probe tissue mechanics. The perturbations are mutually prime frequency components in the range 0.25 to $20 \mathrm{~Hz}$. The calculated input impedance (Zrs) captures the resistive, elastic, and inertive behavior of the lung. These nonparametric data are then fitted to the constant phase model, ${ }^{2}$ where

$$
\begin{gathered}
\mathbb{Z} r s(f)=R+j 2 \pi f I+\frac{G t i-j H t i}{(2 \pi f)^{\alpha}} \\
\alpha=\left(\frac{2}{\pi}\right) \arctan \left(\frac{H t i}{G t i}\right) \\
\eta=\left(\frac{G t i}{H t i}\right)
\end{gathered}
$$

The constant phase model permits a distinction between central and peripheral mechanics. The model includes a parameter for Newtonian resistance (Rn), which is central resistance plus a small and constant chest wall component. The parameter $\mathrm{G}$, or tissue damping, is a frequency-independent parameter that is closely related to tissue resistance, but more accurately reflects the energy dissipated in the tissue. The parameter $\mathrm{H}$ is tissue elastance, which reflects the energy conserved in the tissue. The parameter $\eta$ is tissue hysteresivity, which is derived from the ratio of the energy dissipated over the energy conserved in the tissue $(\eta=\mathrm{G} / \mathrm{H})$. The coefficient of determination provided an evaluation of the model fit and was uniformly greater than $95 \%$ for the data presented.

\section{Pulmonary Corrosion Casting}

After intravascular fixation and lung inflation, the pulmonary circulation was perfused with 2 to $5 \mathrm{~mL}$ of Mercox (SPI, West Chester, Pa) diluted with $20 \%$ methyl methacrylate monomers (Aldrich Chemical, Milwaukee, Wis). After complete polymerization, the tissues were harvested and macerated in 5\% potassium hydroxide followed by drying and mounting for scanning electron microscopy (SEM). ${ }^{3}$ The microvascular corrosion casts were imaged after being coated with gold in an Argon atmosphere with a Philips ESEM XL30 SEM (Eindhoven, Netherlands). Stereo-pair images were obtained with tilt angles from $6^{\circ}$ to $20^{\circ}$. Diameters were interactively measured orthogonal to the vessel axis after storage of calibrated images, using AnalySIS software (version 2.1). The quality of the corrosion casts was controlled by semithin light microscopic sections stained with methylene blue. The corrosion casts demonstrated filling of the whole capillary bed from artery to vein without evidence of extravasation or pressure distention.

\section{Bronchial Corrosion Casting}

After lung inflation and pulmonary intravascular fixation, selective bronchial circulation casting was performed by occluding the pulmonary circulation with $1 \times 10^{6} 15-\mu \mathrm{m}$ microspheres (Bangs Laboratories, Fishers, Ind) via a pulmonary artery infusion. The aortic root was tied off and while back-pressure was being exerted on the microspheres, the bronchial circulation was fixed and cast via retrograde infusions of the descending aorta. A left atriotomy was performed to permit outflow. 


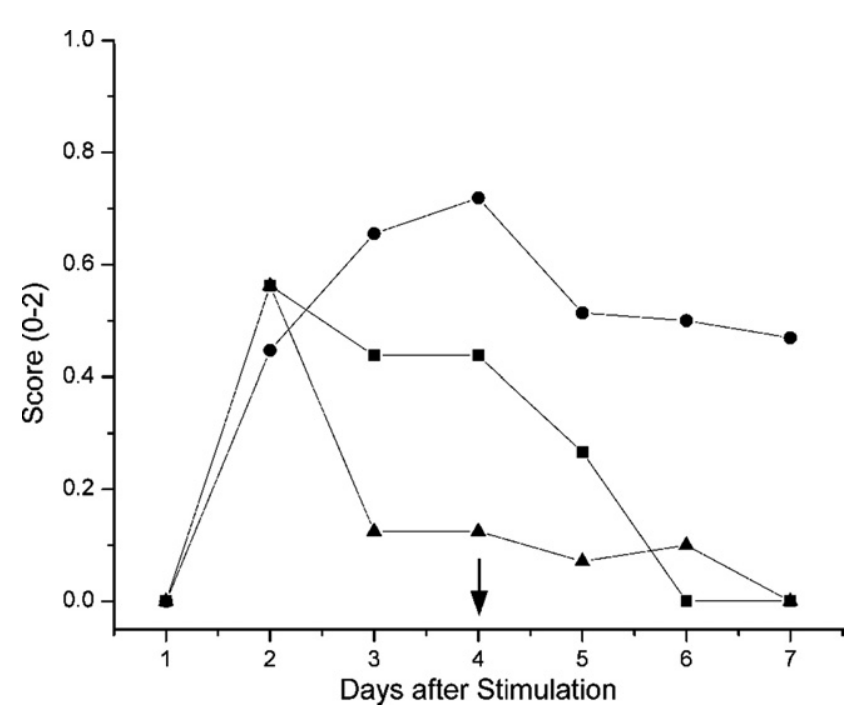

Figure 1. The clinical course of mice after the intratracheal instillation of $15 \mu \mathrm{L}$ of $2.5 \%$ TNBS (N = 209). The assessment of diminished activity level (square), ruffled fur (circle), and respiratory distress (triangle) was performed at 24-hour intervals after the instillation of antigen on day 0 . The scale ranged from 0 (normal) to $\mathbf{2}$ (severe) on all three dimensions with a calculated mean standard deviation for activity level $(S D=0.44)$, ruffled fur $(S D=0.37)$, and respiratory distress $(S D=0.28)$. Subsequent morphologic studies were performed 96 hours after antigen instillation (arrow). TNBS, trinitrobenzene sulfonate.

\section{Results}

\section{Intratracheal Instillation of Antigen}

A model of mononuclear inflammation in the lung was established by intubating anesthetized mice and instilling 15 $\mu \mathrm{L}$ of $2.5 \%$ TNBS into the proximal airways. Unilateral antigen distribution was encouraged by positioning the mice in a right lateral position for 30 minutes before emergence from anesthesia. The clinical response to antigen stimulation, similar to previous work in the skin and colon, demonstrated a reproducible inflammatory response 96 hours after the instillation of antigen (Figure 1). When macroscopically examined at 96 hours, the TNBS-stimulated lungs demonstrated evidence of edema and atelectasis (Figure 2). The inflammation was typically segmental or lobar in distribution within the right lung. Detailed histologic examination demonstrated mononuclear infiltrates in the submucosa of the larger airways, as well as perivascular infiltrates in the smaller pulmonary vessels (Figure 3).

\section{Diminished Tissue Compliance}

To assess the functional consequences of TNBS-induced inflammation, we measured pulmonary mechanics using a forced oscillation ventilator (flexiVent; SciReq, Montreal, Quebec, Canada). The calculated input impedance was fit-

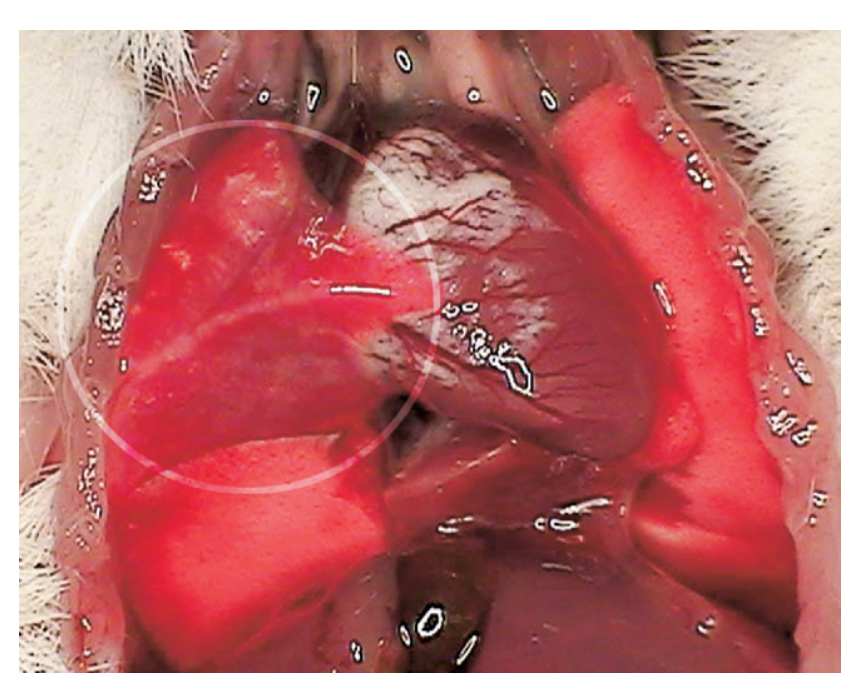

Figure 2. Gross appearance of the lungs 96 hours after the intratracheal instillation of $15 \mu \mathrm{L}$ of $2.5 \%$ TNBS (ventral chest wall removed). Localization of the antigen in the right lung was encouraged with right lateral positioning for $\mathbf{3 0}$ minutes immediately after instillation. At $\mathbf{9 6}$ hours, the right upper lobe was edematous and atelectatic (ring). In contrast, the remaining parenchyma including the right lower lobe and left lung appeared normal. TNBS, trinitrobenzene sulfonate.

ted to the constant phase model of tissue mechanics. ${ }^{2}$ The lung mechanics demonstrated no difference in central airway resistance in the inflammatory lung $(P>.05)$ (Figure $4, A)$. In contrast, peripheral tissue resistance, as reflected by the tissue damping parameter $\mathrm{G}$, was significantly increased compared with control lungs $(P<.00001)$ (Figure $4, B)$. Similarly, the model parameter of tissue elastance $(\mathrm{H})$ and the ratio of tissue damping and tissue elastance $(\eta)$ were
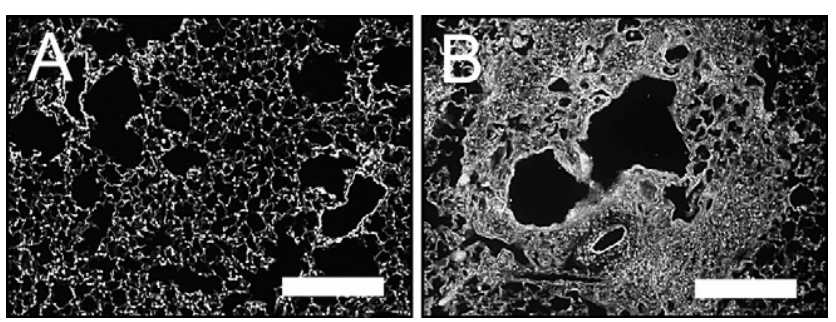

Figure 3. Tissue histologic studies 96 hours after the intrabronchial instillation of $15 \mu \mathrm{L}$ of (A) vehicle control or (B) TNBS antigen (2.5\%). The inflammation in the antigen-stimulated lungs included perivascular mononuclear infiltrates around central and peripheral vessels (bar $=250 \mu \mathrm{m}$ ). The microvessels were identified by intravascular labeling with lipophilic fluorescent tracer, and the mononuclear cells were counterstained with 4,6diamino-2-phenylindole (see Methods). TNBS, trinitrobenzene sulfonate. 
A

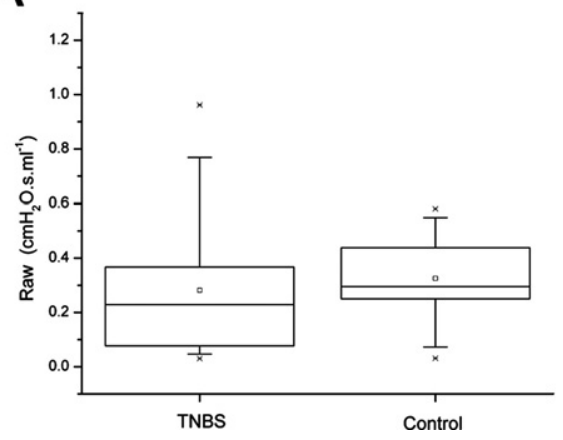

B

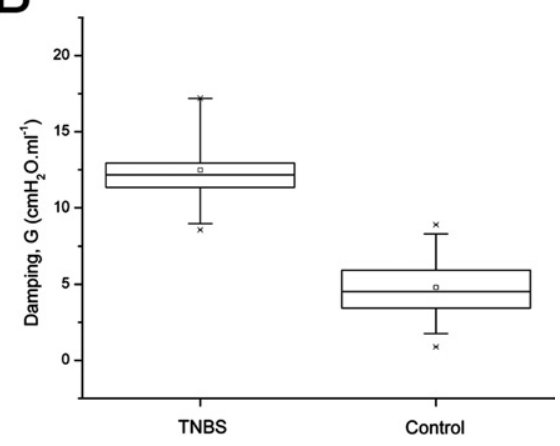

Figure 4. Lung mechanics in mice 96 hours after the intrabronchial instillation of $2.5 \%$ TNBS antigen or PBS control. After endotracheal intubation, input impedance data derived from low-frequency forced-oscillation ventilation (flexiVent, SciReq) was fitted to a multicompartment model. ${ }^{2}$ A, The frequencyindependent airway resistance showed no difference between inflamed and control lungs $(P>.05)(n=5)$. $B$, In contrast, the frequency-dependent peripheral tissue component, expressed as tissue damping $G$, was significantly

greater $(P<.00001)$ than that of the uninflamed control lungs $(n=5)$. The coefficient of determination, a reflection of the adequacy of model fit, was greater than $95 \%$ for all data points. TNBS, trinitrobenzene sulfonate; PBS, phosphate buffered saline.

also significantly different between inflammatory and control lungs $(P<.0001)$.

\section{Structure of Peripheral Pulmonary Circulation}

To determine the effect of peripheral lung inflammation on the murine bronchopulmonary microcirculation, we performed corrosion cast injections of the right side of the heart and descending aorta. Injections of the isolated descending aorta resulted in complete filling of the pulmonary circulation confirming the presence of (1) bronchial arteries and (2) bronchopulmonary interconnections in mice. Three-dimensional SEM of the peripheral lung demonstrated alveolar capillary leak but no evidence of significant structural change (Figure 5).

\section{Structure of Peripheral Bronchial Circulation}

To assess the bronchial circulation in inflammation, we occluded the pulmonary circulation by the injection of $1 \times$ $10^{6}$ microspheres $(15 \mu \mathrm{m})$ into the right side of the heart. Subsequent SEM demonstrated complete filling and functional occlusion of the pulmonary circulation (Figure 6, A). While positive pressure was maintained on the pulmonary microspheres to prevent backflow, corrosion casting injections of the bronchial circulation were performed via the descending aorta. Although the small microsphere-filled pulmonary microvessels were lost during processing, the corrosion-cast bronchial vessels and their interconnection with the pulmonary circulation were identifiable (Figure 6, B). The largest bronchopulmonary interconnections were $20-\mu \mathrm{m}$ vessels demonstrated in the terminal branches of the bronchial arteries (Figure 6, $B$ ).

Also observed in the bronchial circulation were direct connections from bronchial arteries to the alveolar capillary bed. Alveolar capillaries 5 to $10 \mu \mathrm{m}$ in diameter arose directly from bronchial arteries 20 to $50 \mu \mathrm{m}$ in diameter (Figure 7, $A$ and $B$ ). Corrosion casting demonstrated signif- icantly greater perfusion of the alveolar capillary bed in the TNBS-stimulated lung, suggesting adaptive changes in these connections $(P<.05)$ (Figure $8, A$ and $B$ ).

\section{Discussion}

The bronchial circulation plays an important role in such diverse clinical problems as pulmonary embolism, airway reconstructions, and chronic inflammation. In this report, we demonstrated that the intrabronchial instillation of the peptide-hapten trinitrophenol produced a histologic and functional model of peripheral mononuclear inflammation. The model was used to demonstrate the presence of the peripheral bronchial circulation in mice. Morphologic studies demonstrated interconnections between the bronchial and pulmonary circulations at the level of $20-\mu \mathrm{m}$ vessels, as well as structural evidence of increased bronchial perfusion of alveolar capillaries during mononuclear inflammation.

Gross anatomic studies have long demonstrated bronchial arteries supplying bronchial and structural tissues of the lung. ${ }^{1,4}$ The concept of the bronchial arteries also supplying the peripheral lung has been suggested by the preservation of lung structure in the presence of pulmonary arterial obstruction. ${ }^{5-9}$ Defining the structural basis for this "collateral" or "anastomotic" flow, however, has been problematic. Historically, most investigators have used tracer injections and examined the lung in serial tissue sections ${ }^{1,10,11}$ or by chest radiography. ${ }^{8,12,13}$ Whereas the presence of collateral flow was confirmed, these techniques were unable to unambiguously establish the structural basis for these connections.

The development of polymers of sufficiently low viscosity to cast the microcirculation and the use of SEM to define anatomic detail has provided a unique opportunity to identify these structural connections. Previous SEM studies have dem- 

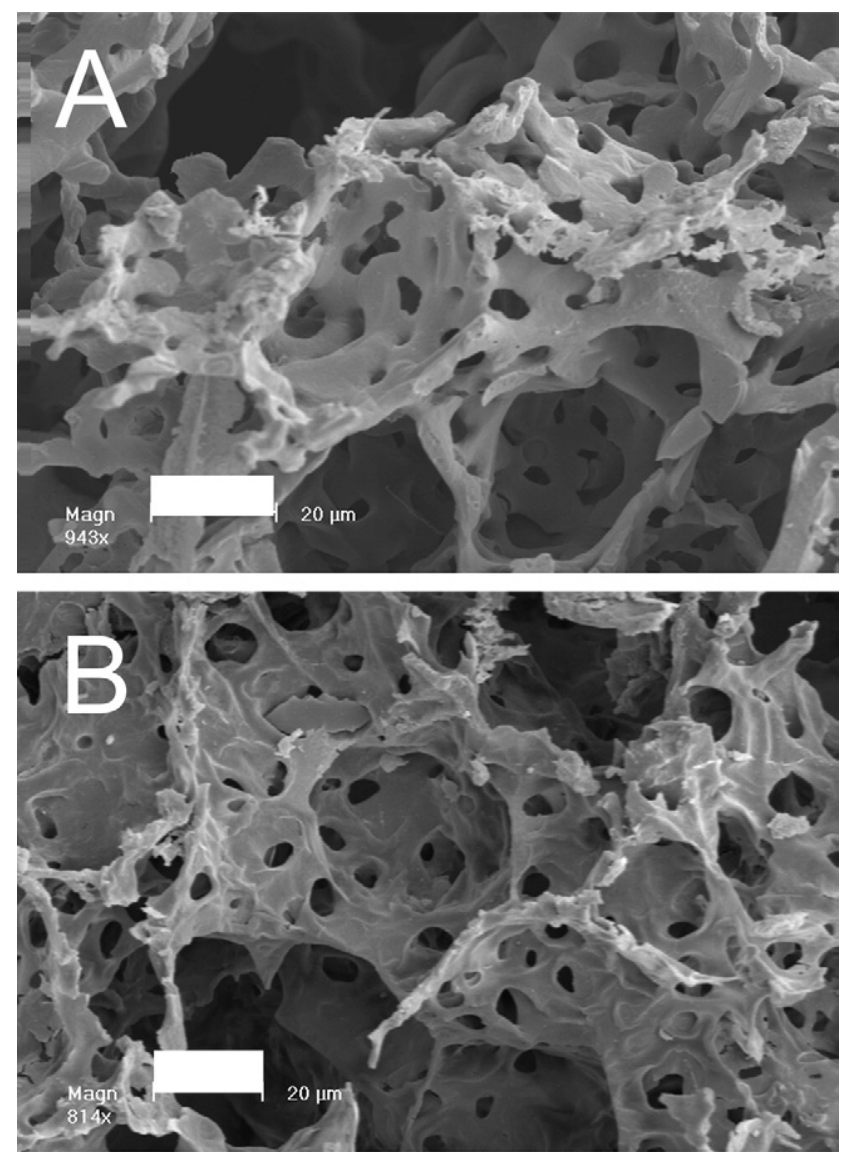

Figure 5. SEMs of the alveolar capillaries in (A) control and (B) TNBS-stimulated mouse lungs. The antigen-stimulated alveoli were imaged 96 hours after the intratracheal instillation of TNBS (bar $=20 \mu \mathrm{m})$. SEM, scanning electron microscopy; TNBS, trinitrobenzene sulfonate.

onstrated extensive collateral flow between the bronchial and pulmonary circulations. In rat studies, Schraufnagel ${ }^{14}$ has reported near complete filling of the pulmonary circulation after injection of the descending aorta, although the site of these interconnections was not identified. In sheep, isolated examples of probable bronchopulmonary vessels have been suggested in dual-circulation SEM studies. ${ }^{15}$ In mice, the structure of the peripheral bronchial circulation remains unclear.

The use of microspheres in our study provided two advantages. First, the $15-\mu \mathrm{m}$ microspheres functionally occluded the pulmonary circulation during competitive corrosion cast injection of the bronchial circulation. The $15-\mu \mathrm{m}$ microspheres were sufficiently small to occlude the distal pulmonary circulation and prevent retrograde filling from the bronchial circulation. Second, the microspheres provided a structural marker for the pulmonary microcirculation. The microspheres allowed us to identify the transition
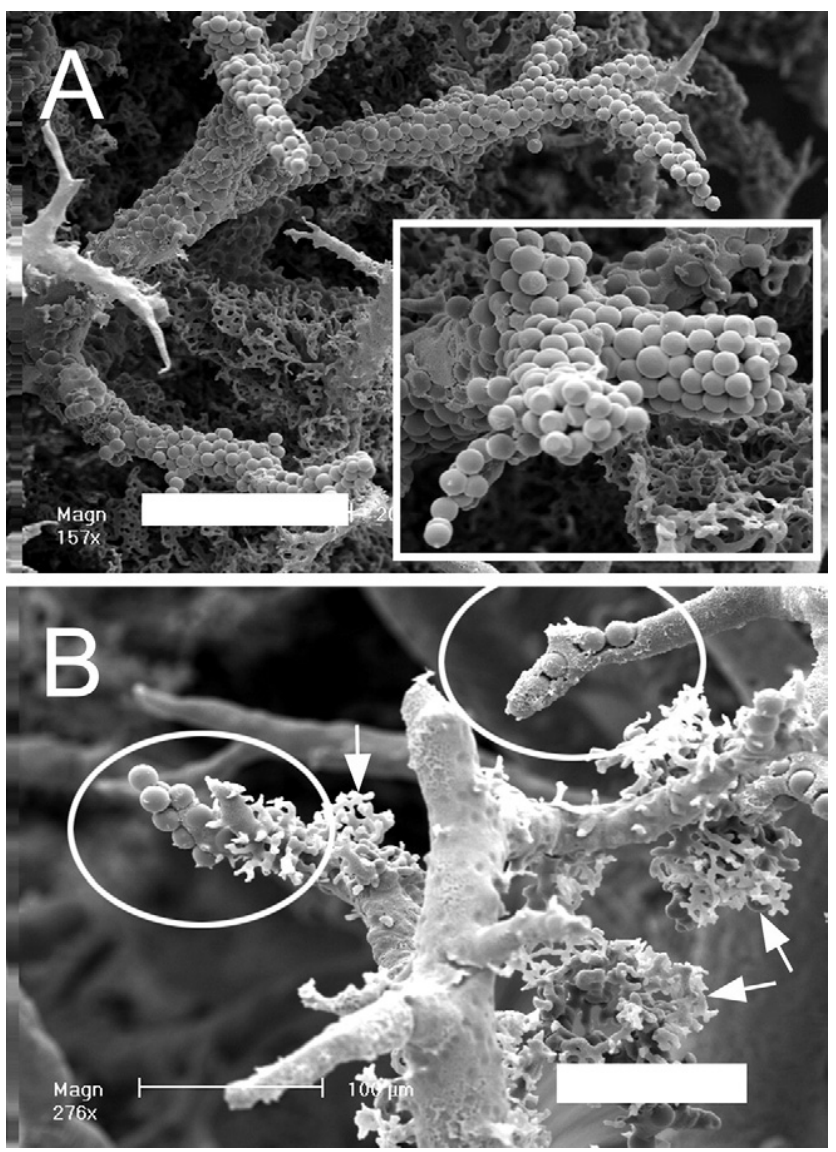

Figure 6. SEMs of the normal peripheral lung after infusion of 15- $\mu \mathrm{m}$ microspheres into the pulmonary circulation and corrosion casting of the bronchial circulation. A, Microspheres injected via the pulmonary artery demonstrate complete occlusion of the central pulmonary vessels (inset) by SEM. B, In contrast to the microsphere-occluded pulmonary circulation, the bronchial circulation was injected with casting material to demonstrate both a bronchial artery and the intercommunications between the pulmonary and bronchial circulations at the level of $\sim 20-\mu \mathrm{m}$ vessels (rings). Note the abbreviated filling of the alveolar capillaries in the terminal branches of the bronchial vessels (arrows). Bar $=100 \mu \mathrm{m}$. SEM, scanning electron microscopy; TNBS, trinitrobenzene sulfonate.

point between bronchial and pulmonary circulations. A potential disadvantage of the small microspheres is that they might have filled the bronchopulmonary connections greater than 15 to $20 \mu \mathrm{m}$. Since the areas filled by microspheres were subsequently undetectable by our morphologic studies, these larger interconnections would not be identified. This possibility should be addressed in future work.

The numerous bronchopulmonary interconnections suggest that, from a morphologic viewpoint, the bronchial and pulmonary circulations merge at the level of $20-\mu \mathrm{m}$ mi- 

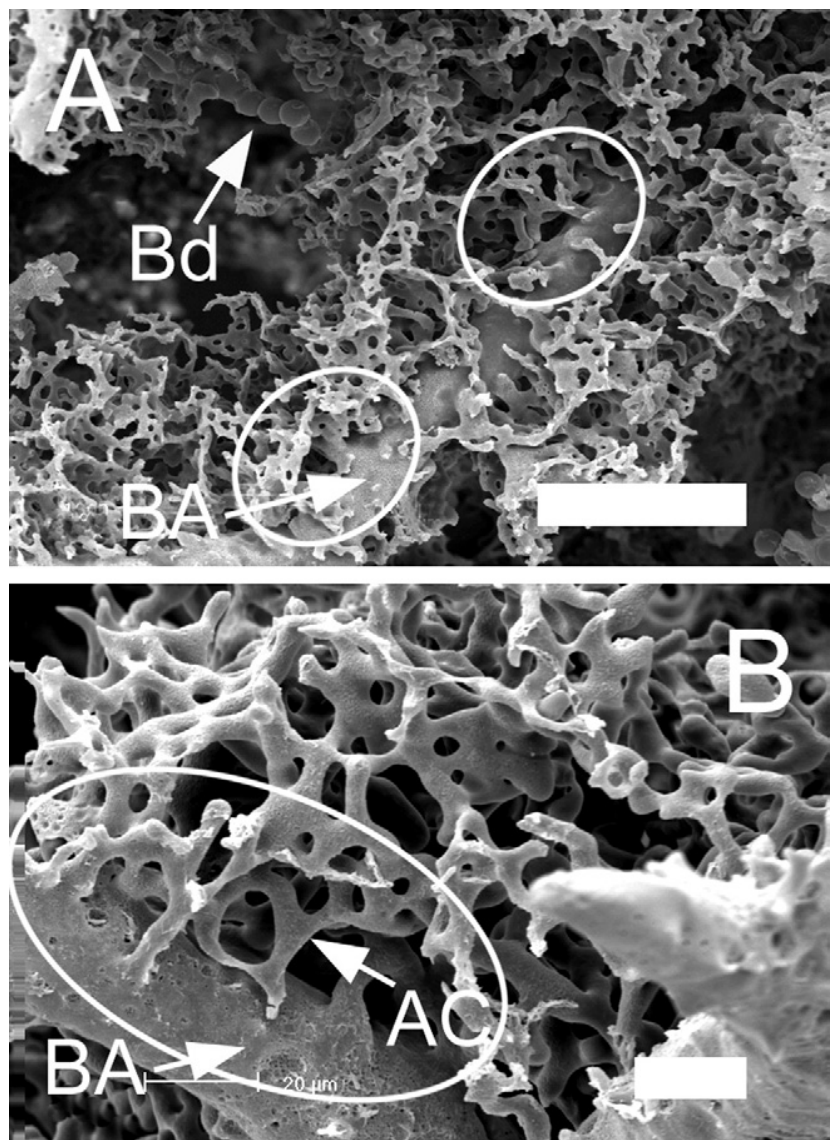

Figure 7. SEMs demonstrating the communication between the bronchial circulation and alveolar capillaries in TNBS-stimulated mice 96 hours after instillation. The bronchial circulation was corrosion cast after pulmonary artery occlusion with $15-\mu \mathrm{m}$ microspheres. A, Bronchial arteries demonstrated serial connections to the alveolar capillaries (rings). Microspheres occluding the pulmonary circulation are visible in the background (bar = $100 \mu \mathrm{m})$. B, The communicating alveolar capillaries arise directly from the bronchial arterioles ( $r i n g)$ (bar $=20 \mu \mathrm{m}$ ). SEM, scanning electron microscopy; TNBS, trinitrobenzene sulfonate; $B d$, Beads; $A C$, alveolar capillaries; $B A$, bronchial artery.

crovessels. The functional consequence of these shared vessels is less clear. In contrast to reports in larger bronchial vessels, ${ }^{16}$ we did not identify sphincter-like structures in the distal bronchial microcirculation. It is likely that the differences in pressure and flow between the systemic and pulmonary circulations - at least in the mouse-dominate the functional consequences of this shared microvascular bed. The pressure and flow relationships in the dual circulations are likely to be similarly relevant to the direct connections observed between the bronchial vessels and the alveolar capillaries.

Finally, the microvascular adaptations observed in the inflamed lung is reminiscent of the bronchial hypertrophy
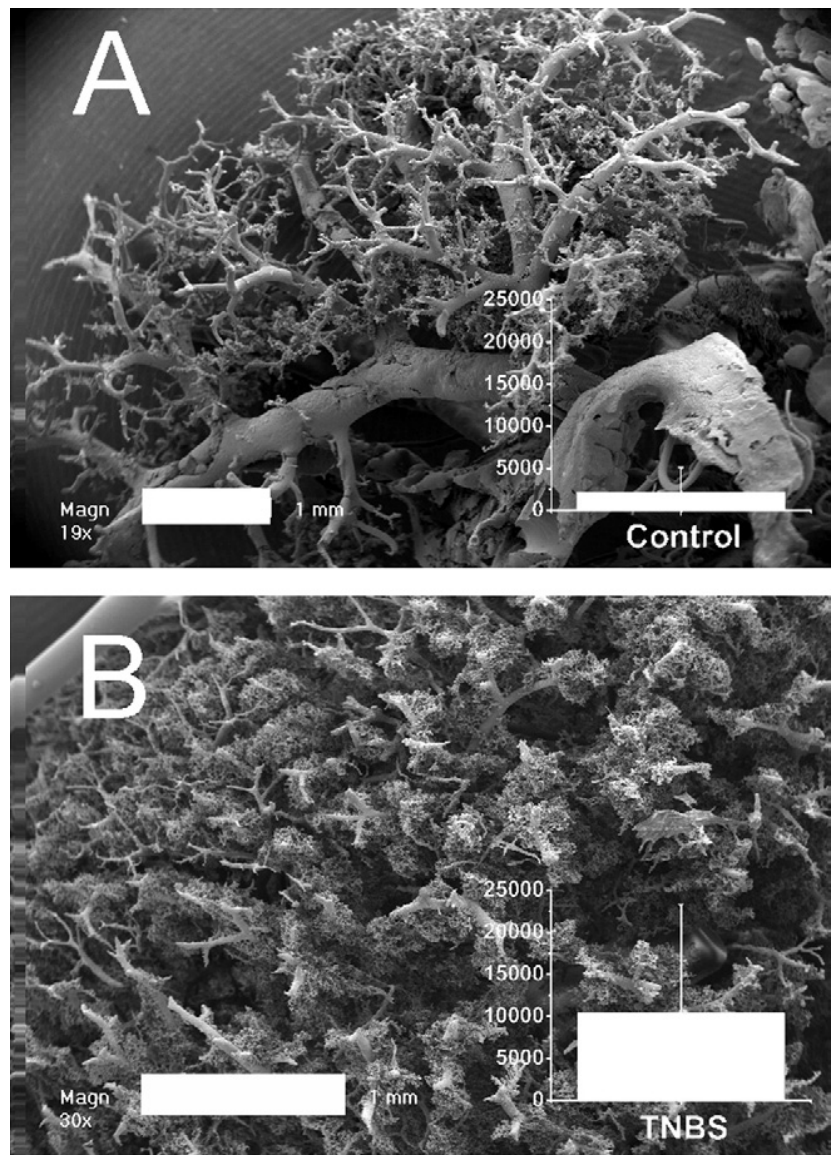

Figure 8. Corrosion cast filling of alveolar capillaries in (A) control and (B) TNBS-stimulated lungs $\mathbf{9 6}$ hours after instillation of antigen. The bronchial circulation was corrosion cast after pulmonary artery occlusion with $15-\mu \mathrm{m}$ microspheres. The number of alveolar tufts per square centimeter in control $(N=3)$ and stimulated $(\mathrm{N}=3)$ mice is shown (insets); error bars equal 1 standard deviation ( $P=.04)$. The variability in the TNBS instillation group is likely related to the inhomogeneous distribution of antigen. TNBS, trinitrobenzene sulfonate.

observed in sheep lung abcesses ${ }^{17}$ and bronchial artery angiogenesis observed in a rat model of monocrotalineinduced injury. ${ }^{18}$ It is also consistent with the clinical findings of bronchial hyperplasia in chronic inflammatory disorders and some neoplasms. ${ }^{13}$ In these examples, it is the systemic bronchial vessels and not the alveolar capillaries capable of adaptive structural changes. The functional adaptations observed in this study suggest the importance of the peripheral bronchial circulation in lung inflammation.

\section{References}

1. Miller WS. The vascular supply of the bronchial tree. Am Rev Tuberc 1925; $12: 87-93$ 
2. Hantos Z, Daroczy B, Suki B, Nagy S, Fredberg JJ. Input impedance and peripheral inhomogeneity of dog lungs. J Appl Physiol. 1992;72: 168-78.

3. Su M, He C, West CA, Mentzer SJ. Cytolytic peptides induce biphasic permeability changes in mammalian cell membranes. J Immunol Methods. 2001;252:63-71.

4. Cauldwell EW, Siekert RG, Lininge RE, Anson BJ. The bronchial arteries. Surg Gynecol Obstet. 1948;86:395-412.

5. Virchow R. Uber die standpunkte in der wissenschaftlichen medicine. Virchows Arch Path Anat. 1847;1:3-19.

6. Karsner HT, Ghoreyeb AA. Studies in infarction: III. The circulation in experimental pulmonary embolism. $J$ Exp Med. 1913;18:507-11.

7. Liebow AA, Hales MR, Bloomer WE, et al. Studies on the lung after ligation of the pulmonary artery. II. Anatomical changes. Am J Pathol. 1950;26:177-95.

8. Jandik J, Endrys J, Rehulova E, Mraz J, Sedlacek J, De Geest H. Bronchial arteries in experimental pulmonary infarction: angiographic and morphometric study. Cardiovasc Res. 1993;27:1076-1083.

9. Tsao MS, Schraufnagel D, Wang NS. Pathogenesis of pulmonary infarction. Am J Med. 1982;72:599-606.

10. Verloop MC. The arteriae bronchiales and their anastomoses with the arteria pulmonalis in the human lung: a microanatomical study. Acta Anat. 1948;5:171-205.

11. Wagenvoort CA, Wagenvoort N. Arterial anastomoses, bronchopulmonary arteries, and pulmobronchial arteries in perinatal lungs. Lab Invest. 1967;16:13-24.

12. Mathes ME, Holman E, Reichert FL. A study of the bronchial pulmonary and lymphatic circulations of the lung under various pathologic conditions experimentally produced. J Thorac Surg. 1932;1:339-62.

13. Wood DA, Miller M. The role of dual circulation in various pathologic conditions of the lungs. J Thorac Surg. 1938;7:649-70.

14. Schraufnagel DE. Microvascular casting of the lung: bronchial versus pulmonary artery filling. Scanning Microsc. 1989;3:575-8.

15. Charan NB, Turk GM, Dhand R. Gross and subgross anatomy of bronchial circulation in sheep. J Appl Physiol. 1984;57:658-64.

16. Tobin CE. The bronchial arteries and their connections with other vessels in the human lung. Surg Gynecol Obstet. 1952;95:741-50.

17. Charan NB, Turk GM, Dhand R. The role of bronchial circulation in lung abscess. Am Rev Respir Dis. 1985;131:121-4.

18. Schraufnagel DE. Monocrotaline-induced angiogenesis. Differences in the bronchial and pulmonary vasculature. Am J Pathol. 1990;137: 1083-90.

\section{Discussion}

Dr Malcolm V. Brock (Baltimore, $M d$ ). The authors present an elegant study using a mouse model to investigate the structural mechanics and the dynamic interactions of the pulmonary and bronchial circulations during chronic inflammation of the lungs. Although the bronchial circulation is known to account for less than $5 \%$ of the blood supply and to provide nourishment to the central airways, very little is known about the structure of the bronchial microcirculation. So little is known, in fact, that there has not even been a demonstration of the existence of bronchial circulation in the mouse before this report.

The authors' mouse model has been well validated in some of their previous work and seems to provide a very reliable, reproducible inflammatory response in the lung, with resulting edema and atelectasis, and histologically there is a very distinct infiltrate that develops. The authors show that the mice clinically become generally lethargic, have trouble breathing, and the smooth appearance of their fur is altered. They further show that the functional consequence of this is noncompliant, stiff lungs. They then take a very innovative approach in using a casting technique, along with a 3-dimensional SEM, to visualize the actual structural connections between the bronchial and pulmonary microcirculations. The results are very clear, dramatic photographs showing what happens to these connections anatomically during a chronic inflammatory response.

I have three questions. First, the casting at 4 days does give us a sort of one-shot view of what happens to these connections. Have you looked at any other time points?

Dr Ravnic. With regard to the timeline, we actually evaluated lungs 2, 4, and 6 days after the induction of pulmonary inflammation. At each of these time points we used histologic examination, corrosion casting, and ventilator measurements. The peak of inflammation consistently occurred at 72 to 96 hours, and this was paralleled by alterations in pulmonary mechanics, as well as an increased flow via the bronchial circulation, as demonstrated by the casts.

Dr Brock. Would you tell us a little bit about the functional and clinical implications of some of this work for our patients, and especially in terms of redefining clinical parameters that we can use for pressure and flow relationships, for example, during a pulmonary embolism?

Dr Ravnic. Because the bronchial and pulmonary microcirculations are so interconnected, it is suggested that alterations in the pulmonary circulation would alter flow and pressure in the bronchial circulation. For example, in inflammatory cases, such as a pulmonary embolism or other types of infection, where the pulmonary circulation autoregulates itself, to minimize ventilation-perfusion mismatching, bronchial flow may be augmented to continue to deliver systemic oxygenated blood as well as inflammatory cells. If we could devise a way to probe the bronchial circulation and its pressure and flow characteristics under these conditions, we might find a correlation with the amount of inflammation taking place in the lung.

Dr Brock. Finally, angiogenesis is known to contribute in many instances in the sort of pathologic damage and repair situation that you see during inflammation. Did you investigate or see any evidence of neovasculature formation in your model, and what about its functional significance?

Dr Ravnic. Although neovascularization is certainly a feasible adaptation during increased perfusion, we have not yet specifically looked at angiogenesis. We have only been able to demonstrate that this functional increase in perfusion occurs during inflammation. However, we do plan to investigate the effects of angiogenesis in the future, as well as any other structural adaptations that might occur in the bronchial circulation.

Dr Stephen G. Swisher (Houston, Tex). Do you know what the underlying cause of these interconnections is? Could it be the alveolar macrophages, and have you looked at any models where the macrophages are depleted?

Dr Ravnic. We have not looked at any models of macrophage depletion. As far as other cells involved in inflammation, we have been studying the role of platelets in altering the structure of the systemic microvascular network. We have not yet studied the role of platelets or microphages in altering the structure of the pulmonary network. 